\title{
Photoluminescence quenching of semiconducting polymer nanoparticles in presence of Au nanoparticles
}

\author{
SANTANU BHATTACHARYYA and AMITAVA PATRA* \\ Department of Materials Science, Indian Association for the Cultivation of Science, Kolkata 700 032, India
}

MS received 17 February 2012

\begin{abstract}
In this report, we have demonstrated the photoluminescence quenching and energy transfer properties of semiconducting polymer nanoparticles, poly ( $N$-vinylcarbazole) (PVK) in presence of different sized Au nanoparticles by steady state and time-resolved spectroscopy. We have described the quenching phenomena by sphere of action static quenching mechanism and both dynamic and static quenching processes are found in these systems. PL quenching values are $\mathbf{2 4 . 2 2 \%}$ and $\mathbf{5 7 . 3 \%}$ for $14 \mathrm{~nm}$ and $18 \mathrm{~nm}$ Au nanoparticles, respectively. It is found that the radiative and nonradiative decay have been modified with the size of Au nanoparticles. PL quenching and shortening of decay time regarding polymer nanoparticles in presence of Au nanoparticles suggest the nonradiative energy transfer process. The values of energy transfer are $6.7 \%, 49.5 \%$ and $53.38 \%$ from PVK polymer nanoparticles to $3 \mathrm{~nm}, 14 \mathrm{~nm}$ and $18 \mathrm{~nm}$ Au nanoparticles, respectively. Using FRET and SET equations we have calculated the average distance of donor PVK polymer nanoparticles and acceptor Au nanoparticles.
\end{abstract}

Keywords. Photoluminescence quenching; semiconducting polymer nanoparticles; Au nanoparticles; time resolved spectroscopy.

\section{Introduction}

Semiconducting conjugated polymeric nanoparticles are very much applicable for their multifunctional activities e.g. imagining, bio-sensing, drugs delivery, photonics and optoelectronics (Yu et al 1995; Kietze et al 2003; Wu et al 2008; Kim et al 2010). The super quenching and hyperefficient energy transfer phenomena of water soluble conjugated polymer molecules attached with Au nanoparticles have been reported previously (Fan et al 2003). Kong et al (2007) reported various photophysical properties of PVK polymer nanoparticles doped by MEH-PPV. It was previously reported that the PL efficiency of Eu-complex varies with changing nature of the semiconducting polymer host (Chowdhury et al 2005). Interactions of different semiconducting polymer nanoparticles with Au nanoparticles have been demonstrated very recently (Bhattacharyya et al 2010). Application of polymer nanoparticles based fluorescence resonance energy transfer using nanoscopic environment is still in the embryonic stage. Further investigations in this field are necessary for an in-depth understanding of the phenomenon for developing new challenging photonic devices.

Dulkeith et al (2002) have shown the modification of radiative and nonradiative properties of lissamine dye in presence of chemically attached different sized Au nanoparticles. They have compared their experimental findings with theoretical results derived from Gerstan-Nitzan model. There are several reports on the photophysical properties and energy transfer phenomena of different semiconducting

\footnotetext{
*Author for correspondence (msap@iacs.res.in)
}

quantum dots and fluorophore in presence of different sized Au nanoparticles (Ghosh et al 2004; Cheng et al 2006; Soller et al 2007). In this report we have analysed the photoluminescence quenching mechanism of PVK polymer nanoparticles in presence of different sized ( $3 \mathrm{~nm}, 14 \mathrm{~nm}$ and $18 \mathrm{~nm}$ ) $\mathrm{Au}$ nanoparticles. The quenching phenomena follows sphere of action static quenching model. According to this model certain fraction of PVK polymer nanoparticles are quenched due to static interaction with Au nanoparticles at their excited state. Rest of the fraction is quenched by collisional mechanism. Therefore, both static and dynamic quenching would be present in such a system. We would like to address a few issues: whether the size of Au nanoparticles influences on the quenching behaviour and on the modification of radiative and nonradiative rates of PVK polymer nanoparticles. Assuming modification of nonradiative decay to be responsible for the resonance energy transfer from PVK to Au nanoparticles, we have demonstrated size dependent variation of resonance energy transfer from PVK to different sized Au nanoparticles by time-resolved spectroscopic study. We have also calculated the distance between Au nanoparticles and PVK polymer nanoparticles by FRET and surface energy transfer mechanism.

\section{Experimental}

\subsection{Materials}

Chloroauric acid $\left(\mathrm{HAuCl}_{4} \cdot 3 \mathrm{H}_{2} \mathrm{O}\right)$ (Loba Chemie), trisodium citrate (Merck), PVK [poly (9-vinylcarbazole)] (Aldrich), 
MPA [3-mercaptopropanoic acid] (Aldrich), sodium hydroxide (Merck), sodium borohydride (Merck) and distilled tetrahydrofuran (Merck) were used in the present study without further purification.

\subsection{Experimental procedure}

PVK conducting polymer nanoparticles were prepared by typical reprecipitation method (Wu et al 2008; Bhattacharyya et al 2010). $1 \mathrm{mg} / \mathrm{ml}$ solution of PVK was prepared in distilled tetrahydrofuran. $200 \mu \mathrm{l}$ of this THF solution was rapidly injected to $20 \mathrm{ml}$ of distilled water under vigorous stirring. After 5-10 min of stirring, it was ultrasonicated for $30 \mathrm{~min}$. Finally, we obtained aqueous suspensions of PVK polymer nanoparticles. Then THF was removed by vacuum evaporation followed by filtration through 0.2 micron filter. The suspensions became stable for 5-7 days.

To prepare smaller sized Au nanoparticles, the well known borohydride reduction method was used. Briefly, we added $500 \mu 1 \mathrm{MPA}$ into $10 \mathrm{ml}$ of distilled water. The aqueous solution turned to acidic. The $\mathrm{pH}$ of this solution became 3-4. $12 \mathrm{ml}$ of this $0.5 \mathrm{M}$ sodium hydroxide solutions was added to this MPA solutions. The resultant $\mathrm{pH}$ of this solution became 8. The concentration of MPA turned to $0 \cdot 17 \mathrm{M}$. Then, $10 \mathrm{ml}$ $0.1 \mathrm{mM}$ aqueous solutions of $\mathrm{HAuCl}_{4}$ and $3 \mathrm{H}_{2} \mathrm{O}$ were taken. $100 \mu \mathrm{l}$ of the previously prepared MPA solution was added to it. Next $0.3 \mathrm{ml} 0.1 \mathrm{M}$ ice cold $\mathrm{NaBH}_{4}$ was dropwise added to it under vigorous stirring. After $10 \mathrm{~min}$ of stirring the solution became brown indicating the formation of small sized Au nanoparticles. A small amount of MPA capped $3 \mathrm{~nm}$ $\mathrm{Au}$ nanoparticles was added to $20 \mathrm{ml}$ of distilled water to maintain Au nanoparticles concentration of $0 \cdot 1 \times 10^{-8} \mathrm{M}$. $200 \mu 1$ THF solution of PVK was rapidly injected to this $0.1 \times 10^{-8} \mathrm{M} 3 \mathrm{~nm}$ Au nanoparticles solution under vigorous stirring followed by $30 \mathrm{~min}$ of ultrasonication. As a result we prepared PVK polymer nanoparticles suspension in 3 nm MPA capped Au colloidal suspensions.

To obtain larger sized Au nanoparticles, well known Frens method (Frens 1973) has been followed. In this method it is possible to control size of the particles by varying [Au (III)/ citrate] ratio during the reduction process. Briefly, three different aqueous solutions of $47.5 \mathrm{ml} \mathrm{HAuCl}_{4}, 3 \mathrm{H}_{2} \mathrm{O}$ (containing $0.01 \mathrm{~g}$ of gold solution) were heated to boiling. Then $2.5 \mathrm{ml}$ of $2 \%$ and $1 \%$ sodium citrate solutions were added to the boiling solutions under vigorous stirring to get $14 \mathrm{~nm}$ and $18 \mathrm{~nm}$ Au nanoparticles, respectively. The colour of the first solution $(2.5 \mathrm{ml} 2 \%$ sodium citrate added) changes from light yellow to deep red through the appearance of bluish gray colour which persists for 5-10 min. For the second solution $(2.5 \mathrm{ml} 1 \%$ sodium citrate added) changes from light yellow to pinkish red through the appearance of this particular bluish gray colour. Then, each of these solutions was allowed to boil for another $20 \mathrm{~min}$. Finally, these solutions were cooled down to room temperature under stirring condition. Thus, we obtained $14 \mathrm{~nm}$ deep red and $18 \mathrm{~nm}$ pinkish red solution of Au nanoparticles. Then appropriate amounts of each of these solutions were added to distilled water to maintain $20 \mathrm{ml} 0 \cdot 1 \times 10^{-8} \mathrm{M}$ Au nanoparticles in each case. Similarly $200 \mu 1$ THF solution of PVK was rapidly injected to each of these solutions under vigorous stirring followed by ultrasonication. Similar vacuum evaporation and filtration through 0.2 micron filter was done. Finally, PVK nanoparticles suspensions in $14 \mathrm{~nm}$ and $18 \mathrm{~nm}$ Au colloidal solutions, respectively were obtained.

The transmission electron microscopy (TEM) images were taken using a JEOL-TEM 2010 transmission electron microscope with an operating voltage of $200 \mathrm{kV}$. Room temperature optical absorption spectra were obtained with an UVvis spectrophotometer (shimadzu). The emission spectra of all the samples were recorded in a Fluoromax-P (HORIBA JOBIN YVON) luminescence spectrometer. For the time correlated single photon counting (TCSPC) measurement, samples were excited at $340 \mathrm{~nm}$ using a picosecond NANO-LED IBH 340. The following expression was used to analyse the experimental time resolved fluorescence decays, $P(t)$ :

$$
P(t)=b+\sum_{i}^{n} \alpha_{i} \exp \left(-\frac{t}{\tau_{i}}\right) .
$$

Here, $n$ is the number of emissive species and $b$ the baseline correction (d.c. offset), and $\alpha_{i}$ and $\tau_{i}$ are the pre-exponential factors and excited state fluorescence lifetimes associated with the $i$ th component, respectively. The average life time, $\langle\tau\rangle$, was calculated from the following formula

$$
\langle\tau\rangle=\sum_{i}^{n} \alpha_{i} \tau_{i}^{2} / \sum_{i}^{n} \alpha_{i} \tau_{i} .
$$

\section{Results and discussion}

\subsection{Morphological analysis}

Figure 1 depicts SEM image of pure PVK polymer nanoparticles. From SEM image it is clear that these polymer nanoparticles are nearly spherical having size distribution range of $60-80 \mathrm{~nm}$. During injection of THF-solution of polymer into water (poor solvents for these kinds of polymer molecules) under stirring condition, the solution is divided into many droplets by strong shearing force. Simultaneously THF molecules quickly diffuse into water to make the polymer chains exposed to water and the polymer chains are coiled up to form polymer nanoparticles (Kong et al 2007). Figure 2 depicts TEM images of different sizes of Au nanoparticles and distribution of size in presence of PVK polymer nanoparticles.

\subsection{Steady state data and quenching mechanism}

Figure 3a shows normalized absorption spectra of different sized Au nanoparticles. Surface plasmon band is not observed for smaller particles $(\sim 3 \mathrm{~nm})$ due to quantum confinement effect and strong dampening of plasmon oscillation. The surface plasmon bands are at $520 \mathrm{~nm}$ and $524 \mathrm{~nm}$ for 


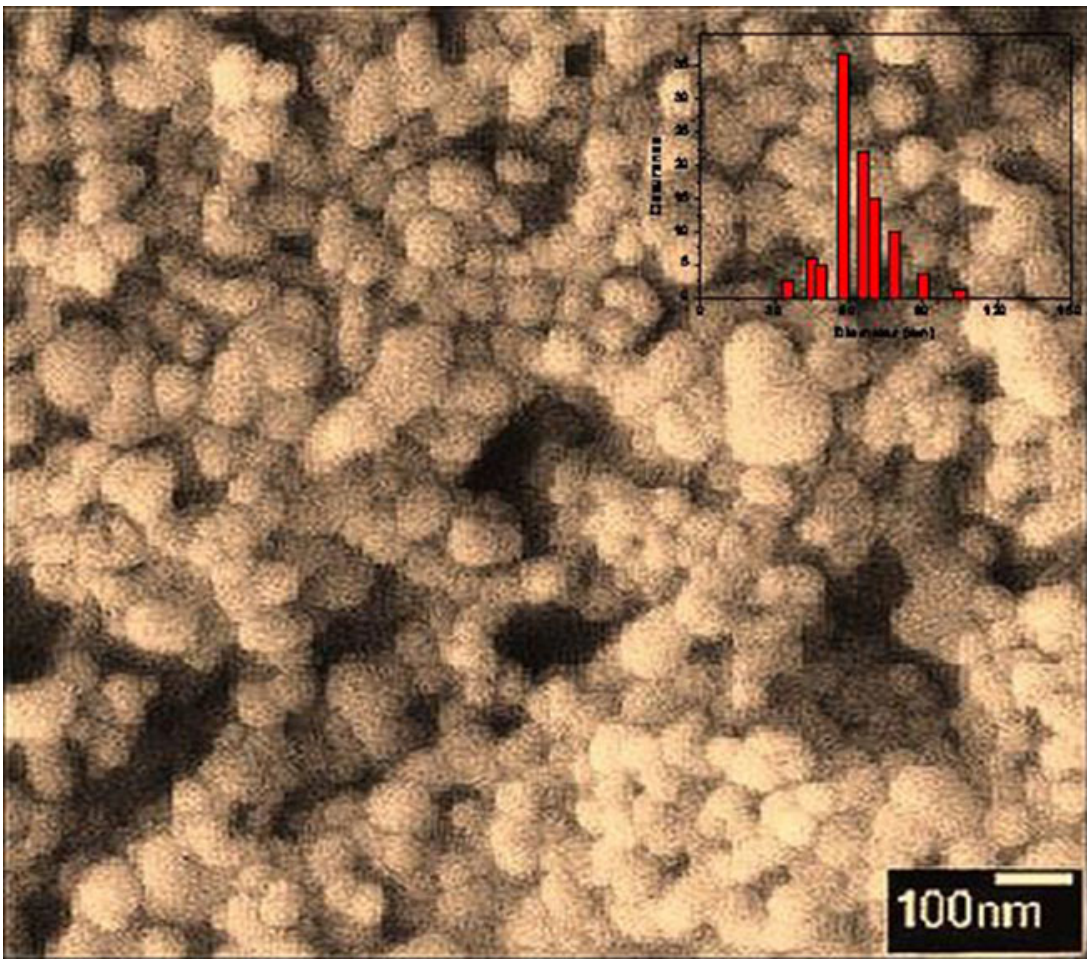

Figure 1. SEM image of PVK polymer nanoparticles.
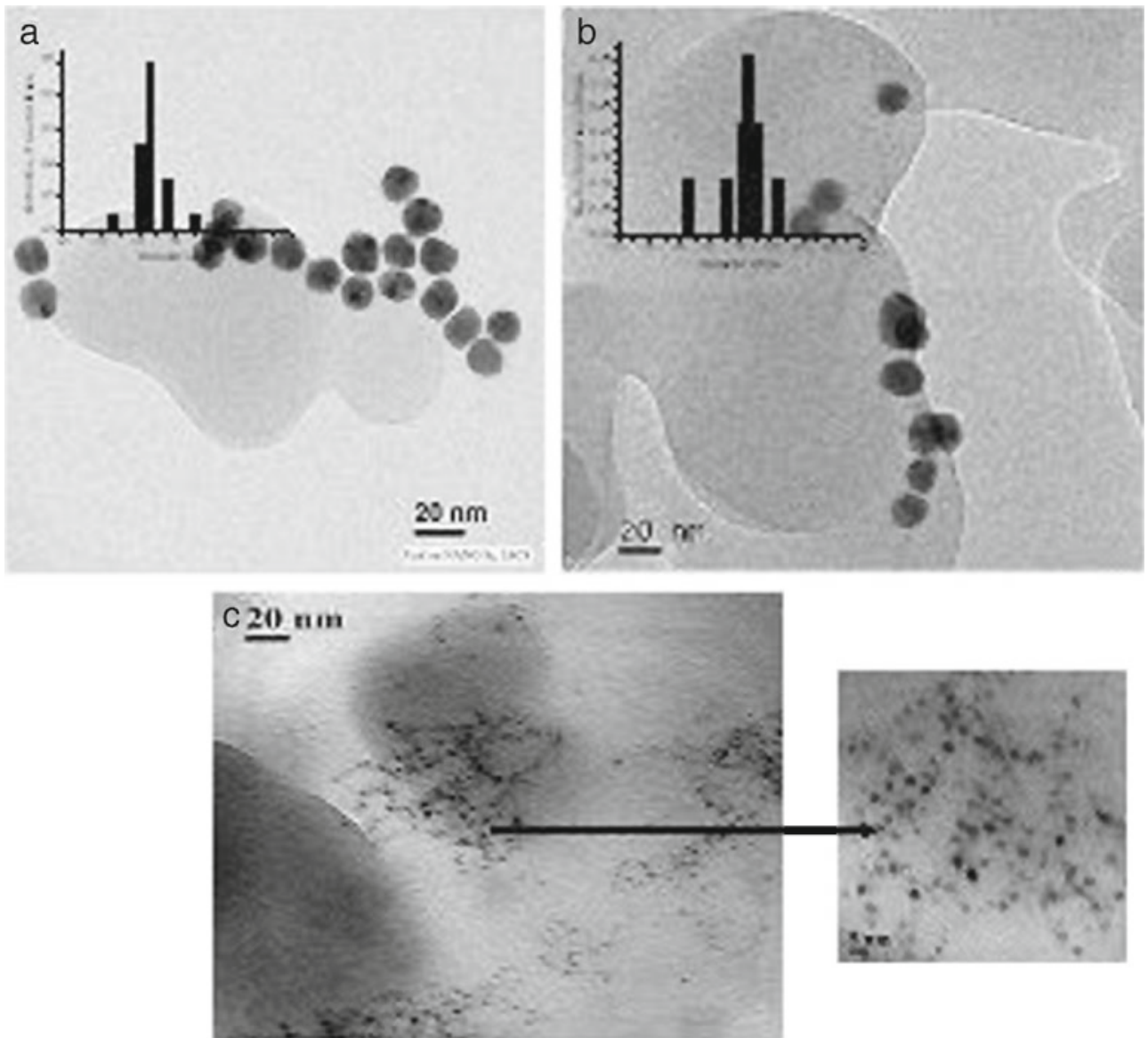

Figure 2. TEM images of PVK polymer nanoparticles in presence of a. $14 \mathrm{~nm}, \mathbf{b} .18 \mathrm{~nm}$ and c. $3 \mathrm{~nm}$ Au nanoparticles. 

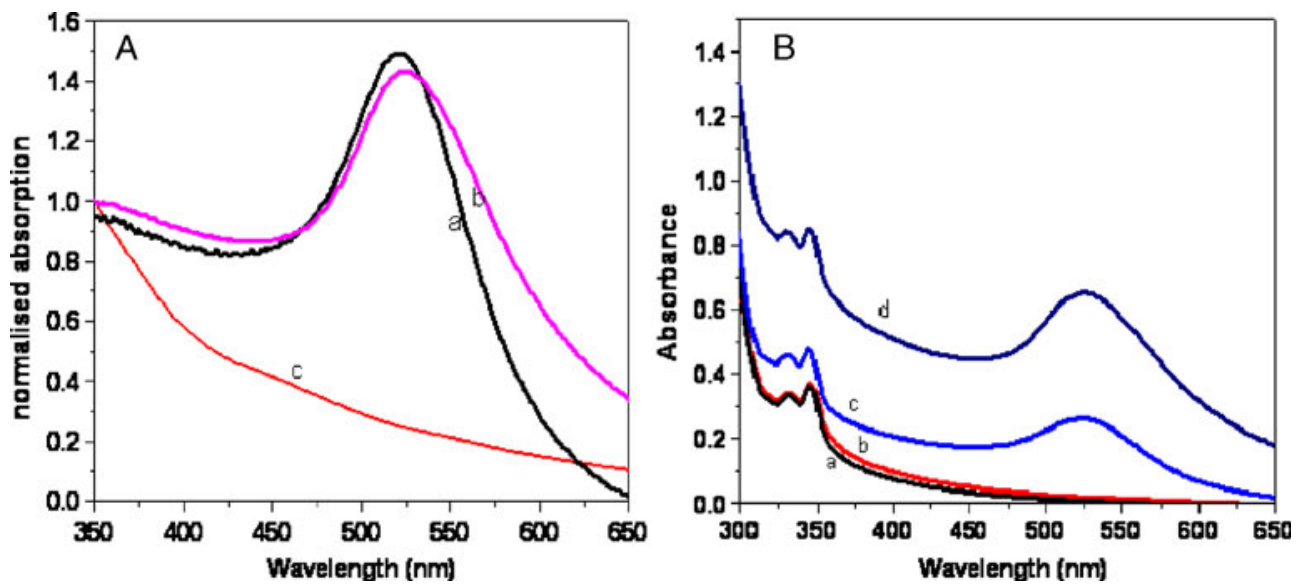

Figure 3. (A) Normalized UV-Vis spectra of a. $14 \mathrm{~nm}$, b. $18 \mathrm{~nm}$ and c. $3 \mathrm{~nm}$ Au nanoparticles and (B) UV-Vis spectra of a. pure PVK, b. PVK+3 nm Au, c. PVK + $14 \mathrm{~nm} \mathrm{Au}$ and d. PVK $+18 \mathrm{~nm} \mathrm{Au}$ (particles concentration of $\mathrm{Au}$ is $0 \cdot 1 \times 10^{-8} \mathrm{M}$ in each case).

$14 \mathrm{~nm}$ and $18 \mathrm{~nm}$ Au nanoparticles, respectively. The extinction coefficient of $3 \mathrm{~nm}$ particle is $0.5 \times 10^{6} \mathrm{M}^{-1} \mathrm{~cm}^{-1}$. For $14 \mathrm{~nm}$ and $18 \mathrm{~nm}$ particles the extinction coefficients are $2.11 \times 10^{8} \mathrm{M}^{-1} \mathrm{~cm}^{-1}$ and $6.28 \times 10^{8} \mathrm{M}^{-1} \mathrm{~cm}^{-1}$ respectively. It matches well with the data described in previous literature (Link and El-Sayed 1999). Figure 3b shows absorption spectra of PVK in absence and presence of different sized Au nanoparticles. The peak position of PVK (almost at $340 \mathrm{~nm}$ ) (Wu et al 2008; Bhattacharyya et al 2010) remains almost constant in presence of different sized Au nanoparticles. The molar extinction coefficient increases with increasing size of Au nanoparticles. The overlap between SPR band of $\mathrm{Au}$ nanoparticles and emission spectra of PVK polymer nanoparticles is very small and the overlap integral cannot be calculated in case of $3 \mathrm{~nm}$ Au nanoparticles. The overlap integrals are $4.67 \times 10^{18} \mathrm{M}^{-1} \mathrm{~cm}^{-1} \mathrm{~nm}^{4}$ and $1.56 \times$ $10^{19} \mathrm{M}^{-1} \mathrm{~cm}^{-1} \mathrm{~nm}^{4}$ for $14 \mathrm{~nm}$ and $18 \mathrm{~nm} \mathrm{Au}$ nanoparticles, respectively. Figure 4 shows PL quenching spectra of PVK polymer nanoparticles in case of different sized $\mathrm{Au}$ nanoparticles. In case of $3 \mathrm{~nm}$ Au nanoparticles there is no quenching. There are $24.2 \%$ and $57.3 \%$ PL quenching for $14 \mathrm{~nm}$ and $18 \mathrm{~nm} \mathrm{Au}$ nanoparticles, respectively. It is to be noted that the concentration of quencher Au nanoparticles remains constant $\left(0.1 \times 10^{-8} \mathrm{M}\right)$ for each case. Thus, size of the quencher Au nanoparticles influences the photoluminescence quenching of PVK polymer nanoparticles. Figure 5a shows Stern-Volmer plot of pure PVK in presence of $14 \mathrm{~nm}$ and $18 \mathrm{~nm}$ Au nanoparticles. We observe a positive deviation of $S-V$ plot in both the cases. The presence of static component in quenching is the cause of this type of positive deviation. The fractional intensity $I_{0} / I$ is given by the product of both static and dynamic quenching. Therefore,

$$
\begin{aligned}
I_{0} / I & =\left(1+K_{\mathrm{s}}[Q]\right)\left(1+K_{\mathrm{sv}}[Q]\right) \\
& =1+K_{1}[Q]+K_{2}[Q]^{2},
\end{aligned}
$$

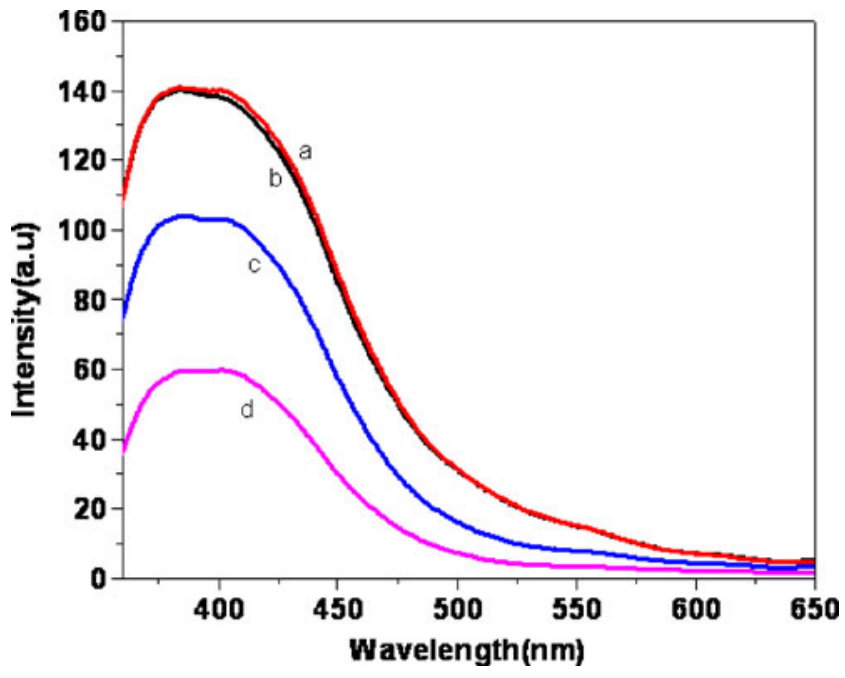

Figure 4. PL spectra of PVK polymer nanoparticles a. without Au nanoparticles and in presence of b. $3 \mathrm{~nm}$, c. $14 \mathrm{~nm}$ and d. $18 \mathrm{~nm}$ Au nanoparticles.

where $K_{1}=\left(K_{\mathrm{sv}}+K_{\mathrm{s}}\right)$ and $K_{2}=\left(K_{\mathrm{sv}} \times K_{\mathrm{s}}\right)$, and $K_{\mathrm{sv}}$ and $K_{\mathrm{S}}$ are the dynamic and static quenching constant, respectively. By applying the above equation, static and dynamic quenching constants are being calculated. The obtained values are found imaginary. Therefore, the sphere of action of static quenching model has been introduced to demonstrate the quenching phenomena properly (Thipperudrappa et al 2007). According to this model, instantaneous or static quenching occurs if the quencher substances are very near to, or in contact with fluorescent molecules at the moment of its excitation. This is explained by the fact that only a certain fraction, $W$, of the excited state is actually quenched by the collision mechanism. Some molecules in the excited state, the fraction $(1-W)$ of which is deactivated almost instantaneously after being formed, because quenchers 

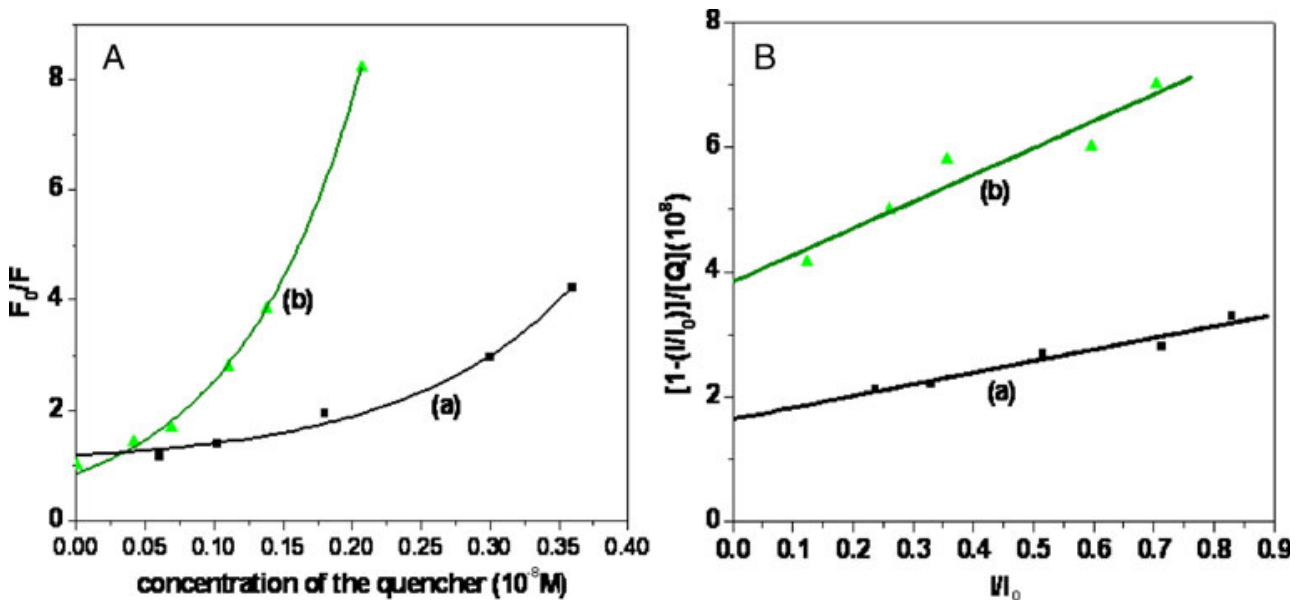

Figure 5. (A) Stern-Volmer plot of PVK polymer nanoparticles in presence of a. $14 \mathrm{~nm}$ and b. $18 \mathrm{~nm}$ Au nanoparticles and (B) $\left[1-\left(I / I_{0}\right)\right] /[Q]$ vs $I / I_{0}$ plot of PVK polymer nanoparticles in presence of a. $14 \mathrm{~nm}$ and b. $18 \mathrm{~nm}$ Au nanoparticles.

happened to be randomly positioned in the proximity at the time the molecules are being excited. Therefore, we have used the following modified form of $S-V$ equation.

$$
\left(I_{0} / I\right)=\left\{1+K_{\mathrm{sv}}[Q]\right\} / W .
$$

The additional factor, $W$, is expressed as

$$
W=\exp (-V[Q]),
$$

where $V$ is the static quenching constant, and it represents an active volume element surrounding in its excited state. Here, $W$ depends on the quencher concentration $[Q]$. At high quencher concentration, the $S-V$ plot deviates from linear character and (4) can be rewritten as

$$
\left[1-\left(I / I_{0}\right)\right] /[Q]=K_{\mathrm{sv}}\left(I / I_{0}\right)+(1-W) /[Q] .
$$

Figure $5 \mathrm{~b}$ depicts plot of $\left[1-\left(I / I_{0}\right)\right] /[Q]$ against $I / I_{0}$ for pure PVK in presence of $14 \mathrm{~nm}$ and $18 \mathrm{~nm}$ Au nanoparticles as quencher. By calculating the slope and intercept, $K_{\mathrm{sv}}$ and $V$ values are calculated. The dynamic and static quenching constants are $1.6 \times 10^{8}$ and $2.2 \times 10^{8} \mathrm{M}^{-1}$, respectively in case of $14 \mathrm{~nm}$ Au nanoparticles. The dynamic and static quenching constants are $4 \times 10^{8}$ and $5.1 \times 10^{8} \mathrm{M}^{-1}$, respectively for $18 \mathrm{~nm} \mathrm{Au}$ nanoparticles. All these data are given in table 1 . Both dynamic and static quenching constants increase with increasing size of Au nanoparticles which might increase the probability of dynamic collisions. In our system the dynamic quenching of PVK polymer nanoparticles by quencher $\mathrm{Au}$ nanoparticles occurred due to long range electronic energy transfer process. And it mainly depends on the overlap between the absorption spectra of different sized Au nanoparticles and broad emission spectra of PVK polymer nanoparticles. As size of the Au nanoparticles increases, overlap integral also increases, resulting in the increment of dynamic quenching constant. Our results have great consistency with the results described by Cheng et al (2006). Besides 'encounter radii' may also
Table 1. PL quenching, static and dynamic quenching constant of PVK nanoparticles in presence of 14 and $18 \mathrm{~nm} \mathrm{Au}$ nanoparticles.

\begin{tabular}{lccc}
\hline & $\begin{array}{c}\text { PL quenching } \\
\text { (\%) of PVK } \\
\text { nanoparticles (in } \\
\text { presence of } \\
0.1 \times 10^{-8} \mathrm{M} \\
\text { quencher) }\end{array}$ & $\begin{array}{c}\text { Dynamic } \\
\text { quenching } \\
\text { constant } \\
\left(\mathrm{M}^{-1}\right)\end{array}$ & $\begin{array}{c}\text { Static } \\
\text { quenching } \\
\text { constant } \\
\left(\mathrm{M}^{-1}\right)\end{array}$ \\
\hline Systems & $24.2 \%$ & $1.6 \times 10^{8}$ & $2.2 \times 10^{8}$ \\
PVK $+14 \mathrm{~nm} \mathrm{Au}$ & $57.3 \%$ & $4 \times 10^{8}$ & $5.1 \times 10^{8}$ \\
PVK $+18 \mathrm{~nm} \mathrm{Au}$ & & & \\
\hline
\end{tabular}

increase with increase in the quencher nanoparticles size. This may also involve in the increment of dynamic quenching constant. It is very much well known that Au nanoparticle is an efficient quencher for fluorescent dye molecules and semiconducting quantum dots. The dynamic quenching constants are in $\sim 10^{6} \mathrm{M}^{-1}$ order of magnitude in these cases (Huang and Murray 2002; Cheng et al 2006). But it should be very high for conjugated polymer systems (Fan et al 2003; Bhattacharyya et al 2010), because excitonic energy diffusion throughout the polymeric chains plays an important role for energy migration and nonradiative resonance energy transfer towards metal nanoparticles. Fan et al (2003) described the superquenching behaviour of cationic fluorescent polymer by Au nanoparticles. The $K_{\mathrm{sv}}$ value reached up to $\sim 10^{11} \mathrm{M}^{-1}$ in their system. They have also showed that quenching constants increase with increase in quencher $\mathrm{Au}$ nanoparticle size. $K_{\mathrm{SV}}$ values in our systems are in $\sim 10^{8} \mathrm{M}^{-1}$ order of magnitude. $K_{\mathrm{SV}}$ values are comparatively high for our systems. But it does not reach as high as $10^{11} \mathrm{M}^{-1}$, because there is neither covalent bonding nor electrostatic interactions between PVK polymer nanoparticles and $\mathrm{Au}$ nanoparticles. On the other hand, greater amount of interaction causes greater amount of excited state complexation 
(described in sphere of action static quenching mechanism) between PVK polymer nanoparticles and Au nanoparticles. Effective perrin radius for sphere of action may also increase with increase in $\mathrm{Au}$ nanoparticle size. Thus, the static quenching constants also increase with increase in size of Au nanoparticles.

\subsection{Time-resolved spectroscopy}

Figure 6 shows decay curves of PVK polymer nanoparticles in absence and presence of different sized Au nanoparticles. In all cases decay curves are fitted by multi-exponential decay. The calculated average decay time of PVK is $0.59 \mathrm{~ns}$. The average decay time of PVK is $0.55 \mathrm{~ns}$ in presence of $0.1 \times 10^{-8} \mathrm{M}$ of $3 \mathrm{~nm}$ Au nanoparticles. The average decay times are $0.3 \mathrm{~ns}$ and $0.27 \mathrm{~ns}$ for PVK nanoparticles in presence of $14 \mathrm{~nm}$ and $18 \mathrm{~nm}$ Au nanoparticles, respectively. The modification of decay time of PVK in presence of different size of $\mathrm{Au}$ nanoparticles is due to both changes in radiative and nonradiative rates. Dulkeith et al (2002) showed a shortening of radiative rate and increment of nonradiative rate of lissamine dye in presence of $\mathrm{Au}$ nanoparticles. We have also observed the change of radiative and nonradiative rates of PVK polymer nanoparticles in presence of different sized Au nanoparticles. All the values are given in table 2. The observed emission lifetime $\left(\tau_{\mathrm{obs}}\right)$ can be combined with fluorescence quantum yield $\left(\phi_{\mathrm{D}}^{0}\right)$ to determine the radiative and nonradiative rates separately for all the cases. The following equations are used to determine the radiative and nonradiative rates (Wu et al 2000).

$$
\begin{aligned}
\kappa_{\mathrm{r}} & =\frac{\phi_{\mathrm{D}}^{0}}{\tau}, \\
\kappa_{\mathrm{nr}} & =\frac{\left(1-\phi_{\mathrm{D}}^{0}\right)}{\tau},
\end{aligned}
$$

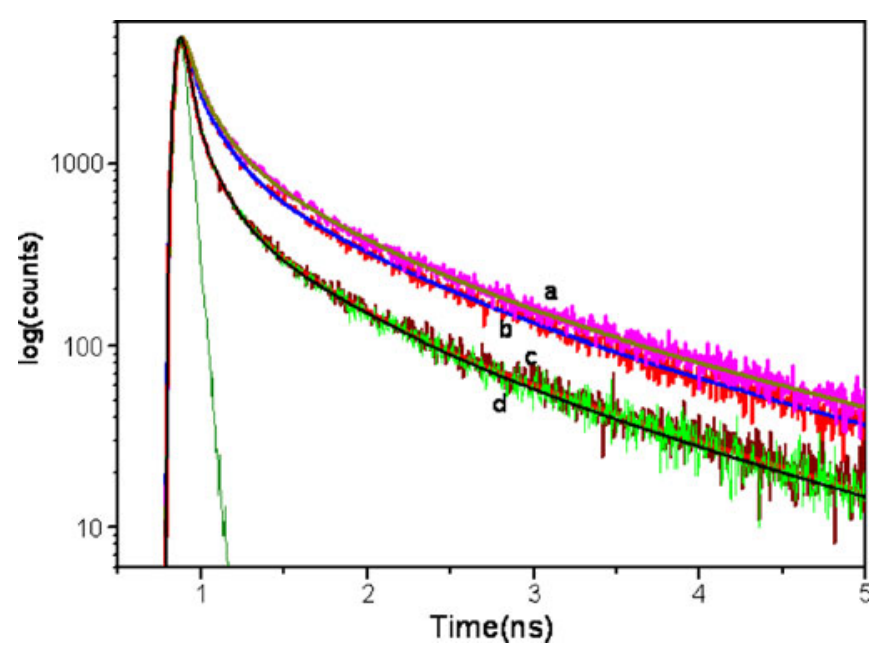

Figure 6. Decay curves of PVK polymer nanoparticles a. without $\mathrm{Au}$ and in presence of b. $3 \mathrm{~nm}$, c. $14 \mathrm{~nm}$ and d. $18 \mathrm{~nm} \mathrm{Au}$ nanoparticles.
Table 2. Quantum yield, radiative and nonradiative decay rates of PVK nanoparticles in presence of different sized $\mathrm{Au}$ nanoparticles.

\begin{tabular}{lrrr}
\hline Systems & $\Phi_{\mathrm{D}}$ & \multicolumn{1}{c}{$\begin{array}{c}\text { Radiative } \\
\text { rate }\left(\mathrm{s}^{-1}\right)\end{array}$} & $\begin{array}{r}\text { Nonradiative } \\
\text { rate }\left(\mathrm{s}^{-1}\right)\end{array}$ \\
\hline PVK & $1.13 \times 10^{-2}$ & $0.19 \times 10^{8}$ & $1.675 \times 10^{9}$ \\
PVK + 3 nm 'Au' & $1.03 \times 10^{-2}$ & $0.234 \times 10^{8}$ & $1.77 \times 10^{9}$ \\
PVK + 14 nm 'Au' & $0.3 \times 10^{-2}$ & $0.11 \times 10^{8}$ & $3.57 \times 10^{9}$ \\
PVK + 18 nm 'Au' & $0.07 \times 10^{-2}$ & $0.025 \times 10^{8}$ & $3.63 \times 10^{9}$ \\
\hline
\end{tabular}

where $\kappa_{\mathrm{r}}, \kappa_{\mathrm{nr}}$ are the radiative and nonradiative rate constants, respectively. Quantum yield of each systems have been calculated by using the following equation

$$
\phi_{\mathrm{D}}^{\mathrm{S}}=\left(F_{\mathrm{S}} \cdot A_{\mathrm{r}} \cdot n_{\mathrm{s}}^{2} \cdot \phi_{\mathrm{D}}^{\mathrm{r}}\right) /\left(F_{\mathrm{r}} \cdot A_{\mathrm{s}} \cdot n_{\mathrm{r}}^{2}\right),
$$

where $\phi_{\mathrm{D}}^{\mathrm{S}}$ is the quantum yield, $F_{\mathrm{S}}$ and $F_{\mathrm{r}}$ are the integrated fluorescence intensity of the sample and reference, respectively. $A_{\mathrm{s}}$ and $A_{\mathrm{r}}$ are the absorbance at the excitation wavelength of the sample and reference, respectively. $\phi_{\mathrm{D}}^{\mathrm{r}}$ is the reference quantum yield. From table 2, radiative and nonradiative rates of pure PVK nanoparticles are $0.19 \times 10^{8} \mathrm{~s}^{-1}$ and $1.675 \times 10^{9} \mathrm{~s}^{-1}$, respectively. In presence of $3 \mathrm{~nm} \mathrm{Au}$ nanoparticles the values are $0.234 \times 10^{8} \mathrm{~s}^{-1}$ and $1.77 \times$ $10^{9} \mathrm{~s}^{-1}$, respectively. Here, both radiative and nonradiative rates have increased in presence of small Au nanoparticles, whereas the radiative rate decreased and nonradiative rate increased in presence of $14 \mathrm{~nm}$ and $18 \mathrm{~nm}$ Au nanoparticles. It is very interesting to note that in presence of smaller $\mathrm{Au}$ nanoparticles there is increment of radiative rate which suggests that small Au nanoparticles have greater field effect originating from inter-plasmonic interaction. Kim et al (2009) described almost the same thing in case of MEHPPV polymer nanoparticles. The increment of nonradiative rate is also very less in case of $3 \mathrm{~nm}$ Au nanoparticles. Therefore, there is no PL-quenching in presence of small $3 \mathrm{~nm} \mathrm{Au}$ nanoparticles. In presence of $14 \mathrm{~nm}$ and $18 \mathrm{~nm}$ Au nanoparticles the nonradiative decay rate are notably increasing. The increase of nonradiative decay rate is due to resonance energy transfer which occurs nonradiatively from donor to acceptor. As the size of the quencher nanoparticles increases the nonradiative decay rate increases which confirms greater amount of resonance energy transfer. The energy transfer efficiency can be calculated by using the following equation.

$$
\phi_{\mathrm{ET}}=1-\frac{\tau_{\mathrm{DA}}}{\tau_{\mathrm{D}}},
$$

where $\tau_{\mathrm{DA}}$ and $\tau_{\mathrm{D}}$ are the decay time of donor fluorescence in presence and absence of acceptor. By using the above equation the calculated energy transfers are $6.7 \%, 49.15 \%$ and $53.38 \%$ for $3 \mathrm{~nm}, 14 \mathrm{~nm}$ and $18 \mathrm{~nm}$ Au nanoparticles, respectively. It reveals that energy transfer efficiency varies with size of Au nanoparticles. 
Table 3. Energy transfer parameter of PVK polymer nanoparticles in presence of $14 \mathrm{~nm}$ and $18 \mathrm{~nm}$ Au nanoparticles.

\begin{tabular}{lccrrrr}
\hline Systems & $J(\lambda) \mathrm{M}^{-1} \mathrm{~cm}^{-1} \mathrm{~nm}^{4}$ & $R_{0}(\AA)$ & $r(\AA)$ & $d_{0}(\AA)$ & $d(\AA)$ & $E(\%)$ \\
\hline $\mathrm{PVK}+14 \mathrm{~nm}$ 'Au' & $4.67 \times 10^{18}$ & 93.16 & 91.42 & 23.5 & 23.03 & 52.54 \\
$\mathrm{PVK}+18 \mathrm{~nm}$ 'Au' & $1.56 \times 10^{19}$ & 113.91 & 111.91 & 23.5 & 22.82 & 53.38 \\
\hline
\end{tabular}

Again, distance of the donor and acceptor is calculated by using FRET method between PVK and Au nanoparticles. Forster distance $R_{0}$ is calculated from the relation

$$
R_{0}=0 \cdot 211\left[\kappa^{2} \eta^{-4} \phi_{\text {donor }} J(\lambda)\right]^{1 / 6} \text { (in angstrom) }
$$

where $\kappa^{2}$ is the orientation factor, $\phi_{\text {donor }}$ the quantum yield of the donor, $J(\lambda)$ the overlap integral between the absorption spectra of acceptor and emission spectra of the donor. $\eta$ is the refractive index of the medium. The calculated $R_{0}$ values are $93.16 \AA$ and $113.91 \AA$ for $14 \mathrm{~nm}$ and $18 \mathrm{~nm} \mathrm{Au}$ nanoparticles, respectively. The calculated average distances between PVK polymer nanoparticles and Au nanoparticles are $91.42 \AA$ and $111.91 \AA$, respectively. It is already reported that FRET based method is restricted on the upper limit of only $80 \AA$ A. We have calculated the distance between donor and acceptor by using surface energy transfer (SET) method. We calculated $d_{0}$ values for each case by using Perrson's model (Chance et al 1978; Yan et al 2005).

$$
d_{0}=\left(\frac{0 \cdot 255 c^{3} \phi_{\mathrm{D}}^{0}}{\omega_{\mathrm{d}}^{2} \omega_{\mathrm{F}} \kappa_{\mathrm{F}}}\right)^{1 / 4}
$$

where $d_{0}$ is the distance at which a fluorophore will display equal probabilities for energy transfer and spontaneous emission. $\phi_{\mathrm{D}}^{0}$ is the quantum efficiency of the fluorophore, $\omega_{\mathrm{d}}$ the frequency of donor angular electronic transition and $\omega_{\mathrm{F}}$ and $\kappa_{\mathrm{F}}$ are the angular Fermi frequency and wave vector of the bulk metal. The $d_{0}$ value is calculated by using quantum yield of PVK as $1.13 \times 10^{-2}$. The calculated $\omega_{\mathrm{d}}^{0}$ value is $4.71 \times 10^{15} \mathrm{~s}^{-1} . \omega_{\mathrm{F}}$ and $\kappa_{\mathrm{F}}$ are $8.4 \times 10^{15} \mathrm{~s}^{-1}$ and $1.2 \times 10^{8} \mathrm{~s}^{-1}$ and $c$ the velocity of light. The calculated $d$ values are 23.03 $\AA, 22.82 \AA$ in case of $14 \mathrm{~nm}$ and $18 \mathrm{~nm} \mathrm{Au}$ nanoparticles, respectively. It is already reported that in case of quencher Au nanoparticle SET model is more appropriate due to the various limitations of FRET (Jennings et al 2006). Therefore, distance calculated from SET model should be more appropriate in our system. All these values are given in table 3.

\section{Conclusions}

Here, we showed the Au nanoparticle size dependent study on photoluminescence quenching and energy transfer of PVK polymer nanoparticles. Both static and dynamic quenching constants are increased with increasing size of $\mathrm{Au}$ nanoparticles. Radiative and nonradiative rates of PVK have been modified in presence of Au nanoparticles. Analysis suggests that the modification of nonradiative rate indicates the resonance energy transfer between polymer nanoparticles and Au nanoparticles. The percentage of energy transfers are $6.7 \%, 49.5 \%$ and $53.28 \%$ for $3 \mathrm{~nm}, 14 \mathrm{~nm}$ and $18 \mathrm{~nm}$ Au nanoparticles, respectively. Using surface energy transfer model, the calculated average distances between PVK and Au nanoparticles are 22.03 $\AA$ and $23.5 \AA$ for $14 \mathrm{~nm}$ and $18 \mathrm{~nm}$ Au nanoparticles, respectively. Therefore, photoluminescence quenching, radiative, nonradiative decay and energy transfer efficiency of semiconducting polymer nanoparticles depend on Au nanoparticles size.

\section{Acknowledgements}

The CSIR and "Ramanujan Fellowship" are gratefully acknowledged for financial support. One of the authors (SB) thanks CSIR for awarding a fellowship.

\section{References}

Bhattacharyya S, Sen T and Patra A 2010 J. Phys. Chem. C114 11787

Chance R, Prock A and Silbey R 1978 Adv. Chem. Phys. 601

Cheng P H, Silvester D, Wang G, Kalyuzhny G, Douglas A and Murrary R W 2006 J. Phys. Chem. B110 4637

Chowdhury P S, Saha S and Patra A 2005 Chem. Phys. Lett. 405393

Dulkeith E et al 2002 Phys. Rev. Lett. 89203002

Fan C H, Wang S, Hong J W, Bazan G C, Plaxco K W and Hegger A J 2003 Proc. Natl. Acad. Sci. U.S.A. 1006297

Frens G 1973 Nature 24120

Ghosh S K, Pal A, Kundu S, Nath S and Pal T 2004 Chem. Phys. Lett. 395366

Huang T and Murray R W 2002 Langmuir 1237077

Jennings T L, Singh M P and Strouse G F 2006 J. Am. Chem. Soc. 1285462

Kietze T, Nehrer D, Landfester K, Montenegro R, Guntur R and Scherf U 2003 Natl. Mater. 2408

Kim M S et al 2009 ACS Nano 31329

Kim S, Lim C K, Na J, Lee Y D, Choi J, Leary J F and Kwon I C 2010 Chem. Commun. 461617

Kong F, Sun Y M and Yuan R K 2007 Nanotechnol. 18265707

Link S and El-Sayed M A 1999 J. Phys. Chem. B103 8410

Soller T et al 2007 Nano Lett. 71941

Strouse G F 2005 J. Am. Chem. Soc. 1273115

Thipperudrappa J, Biradar D S and Hanagodimath S M 2007 J. Lumin. 12445

Wu F, Zhang J Z, Kho R and Mehra R K 2000 Chem. Phys. Lett. 330237

Wu C, Bull B, Szymanski C, Christensen K and McNeill J 2008 ACS Nano 112415

Yan C S et al 2005 J. Am. Chem. Soc. 1273115

Yu G, Gao J, Hummelen J C, Wudl F and Hegger A J 1995 Science 2701789 